\title{
Research on the performance of multi-user detection algorithm based on serial and threshold in the IoT
}

\author{
Jingqiu Ren ${ }^{1[0000-0002-4240-1748]}$, Jianpeng Zhang ${ }^{1}$ \\ Zonglin $\mathrm{Gu}^{1}$, Guanghua Zhang ${ }^{1 *[0000-0002-9050-1284]}$, Weidang $\mathrm{Lu}^{2}$ \\ ${ }^{1}$ Northeast Petroleum University, Daqing 163318, China; \\ ${ }^{2}$ Zhejiang University of Technology, Hangzhou 310014, China; \\ dqzgh@nepu.edu.cn
}

\begin{abstract}
In the 5G system for the Internet of Things (IoT), the detection and identification of a large number of IoT users or devices has become a key issue in the research of IoT technology. In the multi-user detection algorithm based on $5 \mathrm{G}$ technology, due to the slow convergence speed and high complexity of the traditional algorithm, people began to consider the conditions of adding serial and threshold to study, in order to improve the performance of multi-user detection in the IoT based on 5G. In this paper, based on the research of Maximum logarithm Message Passing Algorithm (Max-log-MPA) based on threshold, the serial condition setting is added to analyze the performance of Max-log -MPA algorithm based on serial and threshold (S-T-Max-log-MPA) and Maxlog-MPA algorithm based on threshold (T-Max-log-MPA), so as to obtain a better multi-user detection algorithm based on $5 \mathrm{G}$ in the Internet of things. The simulation results show that the S-T-Max-log-MPA algorithm has lower bit error rate and better performance in the experiment, which is more suitable for multi-user detection in the Internet of things based on $5 \mathrm{G}$.
\end{abstract}

Keywords: Internet of things, sparse code multiple access technology, MPA algorithm, bit error rate, serial

\section{Introduction}

In recent years, with the rapid development of big data, cloud computing, smart city, etc., the network is facing the growing demand of high capacity, high transmission rate and low delay, and the Internet of Things (IoT) industry has entered a stage of rapid development ${ }^{[1]}$. Especially with the rapid development of $5 \mathrm{G}$ technologies, the application of the IoT breaks the established concept of people to people interconnection of the existing mobile network, and realizes the real IoT, people to people and people to things ${ }^{[2]}$, bringing great convenience to people's production and life. In the application of IoT based on 5G, the number of users is usually large. How to use multi-user detection to solve such problems as reducing the cost of machine terminals, resource allocation and low-cost IoT terminal coverage has become an important research issue ${ }^{[3]}$. 
Sparse Code Multiple Access (SCMA) is one of the core technologies of 5G. It is a new orthogonal multiple access technology using sparse codebook, and it is the extension of Low Density Signal (LDS) ${ }^{[4]}[5]$. When detecting the accuracy of data and dealing with multi-user access problems, SCMA multiple access technology realizes the capacity of more users under the same number of resources, increases the overall throughput of the network, and realizes massive connections through code field sparse expansion and non-orthogonal superposition. In addition, LDS integrates the modulation idea of sparse spread spectrum and high-dimensional modulation technology, mapping the bit data stream in the link to the multi-dimensional codeword in the preset codebook, to solve the system overload problem caused by a large number of data connections ${ }^{[6] .}$ However, in the existing SCMA system, when the Message Passing Algorithm (MPA) performs multi-user detection of the received signal ${ }^{[7]}$, the calculation complexity of the algorithm is too high and the transmission accuracy is poor, so it is difficult to apply it to the actual decoding process ${ }^{[8]}$. Therefore, the accuracy of multi-user detection algorithm in the transmission of information is still one of the important challenges in the multi-user detection of the IoT $^{[9]}$. In reference [10], it is described that in the context of IoT communication, compressed sensing is used for multi-user detection. Many studies assume that the channel information is known, and in practical applications, channel estimation is also needed which improves the complexity of the system. The Maximum logarithm Message Passing Algorithm (Maxlog-MPA) based on log domain and the algorithm based on serial MPA (S-MPA) are proposed in reference[11] [12], it reduces the computational complexity, but the system loss is large. In reference [13], an improved multi-user detection algorithm based on partial edge is proposed. Although the complexity of the algorithm is reduced, the BER performance is also reduced. In reference [14], an improved serial scheduling based MPA (ISS-MPA) detection scheme is proposed. Although it can maintain good bit error rate performance, the complexity effect of the detection algorithm is not particularly good. In reference [15], a threshold based MPA algorithm is proposed, which uses a threshold to control the algorithm, so as to reduce the complexity of detection algorithm. In reference [16], an algorithm is proposed to reduce the decoding complexity of SCMA by introducing a weighting factor instead of the iterative process of MPA algorithm. In reference [17], a threshold-based Max-log-MPA low complexity multiuser detection algorithm is proposed. The influence of the change of signal-to-noise ratio on complexity is analyzed to reduce the complexity of multiuser detection better.

Among the above algorithms, a new method is proposed to compare with the traditional MPA algorithm, so as to prove the superior performance of the proposed algorithm and analyze the performance of each algorithm. Therefore, based on the research of Max-log-MPA algorithm based on threshold, this paper adds serial condition setting to analyze the performance of the Max-log-MPA algorithm based on serial and threshold (S-T-Max-log-MPA) and the Max-log MPA algorithm based on threshold (T-Max-log-MPA), so as to select an optimal multi-user detection algorithm to solve the problem of multi-user detection in the application of IoT based on 5G. 


\section{SCMA system model}

Sparse code multiple access(SCMA) is a non-orthogonal access method based on code domain ${ }^{[18]}$. At the transmitter, each user has its own codebook ${ }^{[19]}$, and the transmitter consists of two parts: multidimensional modulation and sparse spread spectrum [20-22]. Sparse spread spectrum plays an important role in transmission. The information bits of different users are mapped into multi-dimensional codewords after channel coding, and then are non-orthogonal stacked on the same resource block by sparse spread spectrum.

The SCMA uplink model consists of transmitter, transmission channel and receiver, as shown in Figure 1. It can be seen that in the multi-user SCMA system, different binary bitstreams are mapped to the codewords of SCMA multi-dimensional codebook by different users through different codebooks. Assuming that the number of users and codebook of IoT is $\mathrm{J}$, and the codebook length is $\mathrm{K}(\mathrm{J}>\mathrm{k})$, then the users of IoT $u_{j}\left(u_{1}, u_{2}, \cdots, u_{J}\right)$ get binary bit data stream $b_{j}\left(b_{1}, b_{2}, \cdots, b_{J}\right)$ after source coding and channel coding. When entering the SCMA encoder transmission, the $j$-th user's bit stream is mapped to the orthogonal subcarrier through the codebook. The mapping process can be expressed as $f: B^{\log _{2} M} \rightarrow \chi^{[23][24]}$, B represents the set of binary numbers, and $\chi$ is the user's codebook. Because the channels of each layer of the upper link are different, the channel factor is different. Suppose the channel factor is $\mathrm{h}_{\mathrm{j}}\left(\mathrm{h}_{1}\right.$, $\left.\mathrm{h}_{2}, \ldots, \mathrm{h}_{\mathrm{J}}\right)$.

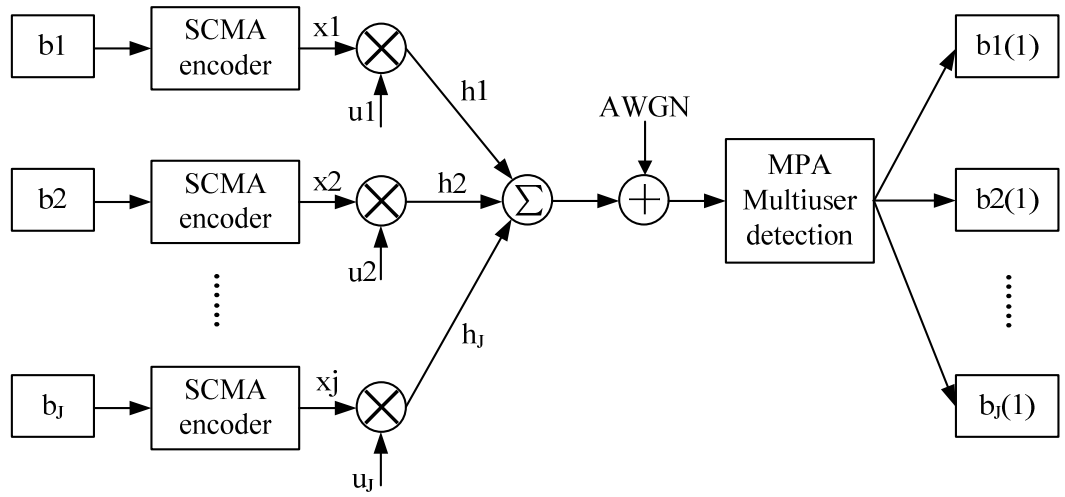

Figure1 uplink SCMA communication system model

$\mathrm{M}$ in $B^{\log _{2} M}$ represents the size of codebook. Assuming that the user node data is divided into several groups according to a-bit, the size $\mathrm{M}$ of codebook is:

$$
\mathrm{M}=2^{\mathrm{a}}
$$

First, define an overload factor $\lambda=J / K$, assume that there are 6 user nodes and 4 time-frequency resource blocks, as shown in Figure 2. It can be seen that at this time, the overload factor $\lambda=1.5$, in other words, the system has $150 \%$ overload capacity. As shown in Figure 3, user 1, .., user6 represents six different user nodes. The bit information of these six user nodes is mapped to the codewords of different codebooks, and each user node has and only has one codebook corresponding to it. 


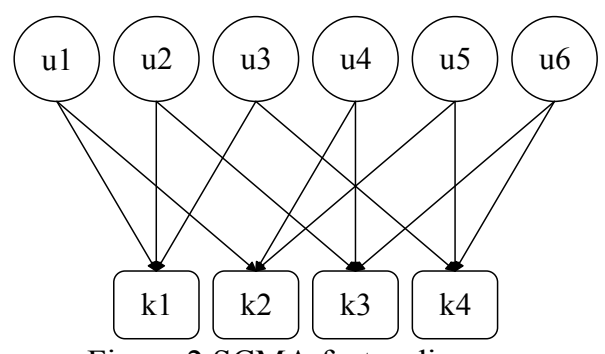

Figure 2 SCMA factor diagram

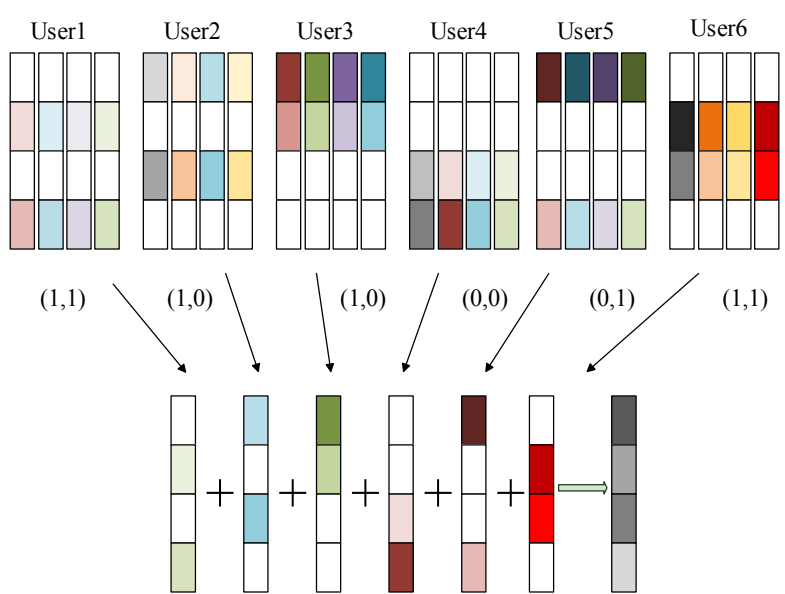

Figure 3 SCMA coding principle

From figure 2 and Figure 3, it can be seen that the area where element 0 is located is a white area, and the area where element 1 is located is a colorful area, it means that in user1, orthogonal time-frequency 1, 3 resources transmit signals, 2, 4 resources do not transmit signals, and so on, the following matrix can be obtained:

$$
F_{4 \times 6}=\left[\begin{array}{cccccc}
0 & 1 & 1 & 0 & 1 & 0 \\
1 & 0 & 1 & 0 & 0 & 1 \\
0 & 1 & 0 & 1 & 0 & 1 \\
1 & 0 & 0 & 1 & 1 & 0
\end{array}\right]
$$

Assuming that the time of each IoT user is synchronous, the signal received by the base station is the weighting of all signals:

$$
y=\sum_{j=1}^{J} \operatorname{diag}\left(\mathrm{h}_{j}\right) x_{j}+n
$$

Where, $x_{j}=\left[x_{1, j}, x_{2, j}, \cdots, x_{K, j}\right]^{T}$ is the codeword sent by user $\mathrm{j} . \mathrm{h}_{\mathrm{j}}=\left[\mathrm{h}_{1}, \mathrm{~h}_{2}, \ldots, \mathrm{h}_{\mathrm{K}}\right]^{\mathrm{T}}$ represents the receiver channel vector, which is the channel gain matrix. $\mathrm{n}$ represents the Gaussian white noise in the transmission channel $n \sim C N\left(0, \sigma^{2} I\right)$. In this case, the signal received by the time-frequency resource at $\mathrm{K}$ can be expressed as: 
$y_{k}=\sum_{j=1}^{j} h_{k}, x_{k}^{j}+n_{k}$. Because of its sparsity, the code word conflict at time-frequency resource $\mathrm{K}$ will be greatly reduced ${ }^{[25]}$.

\section{T-Max-log-MPA algorithm}

The T-Max-log-MPA algorithm is based on the Threshold based MPA algorithm (T-MPA), adding the judgment of the necessary conditions for the stability of user nodes. Before updating the message, first judge whether the user information nodes meet the necessary conditions for the stability of user nodes, and then judge whether they pass the threshold conditions. Only when users who meet the threshold conditions and pass the necessary condition of user node stability can be decoded in advance.

In the SCMA iteration process, if the user node $u_{j}$ in the factor graph is in the same position in the $\mathrm{i}$-th cycle iteration process, that is, the $\mathrm{i}$-th iteration is equal to the result of the i'-th iteration ${ }^{[26]}: \arg \max _{1 \leqslant m \leqslant M} q^{i}\left(\chi_{j, m}\right)=\arg \max _{1 \leqslant m \leqslant M} q^{i^{\prime}}\left(\chi_{j, m}\right), i<i^{\prime}<I_{\max }$, This indicates that the user $u_{j}$ node is stable. Therefore, in the i-th iteration and the (i+1)th iteration, the same position of the largest element in the codeword credibility vector is a necessary condition for the stability of the user node,

$\arg \max _{1 \leqslant m \leqslant M} q^{i}\left(\chi_{j, m}\right)=\arg \max _{1 \leqslant m \leqslant M} q^{i+1}\left(\chi_{j, m}\right)$.

The algorithm can be divided into three steps:

Step 1: receive condition initialization:

$$
\begin{gathered}
I_{c_{k} \rightarrow u_{j}}^{0}\left(x_{j}\right)=\frac{1}{M} \\
I_{c_{k} \rightarrow u_{j}}^{t}\left(x_{j}\right)=\sum_{\sim x_{j}}\left\{\frac{1}{\sqrt{2 \pi} \delta} \exp \left(-\frac{1}{2 \delta^{2}}\left\|y_{k}-\sum_{v \in \xi_{k}} h_{k, v} x_{k, v}\right\|^{2} \times \prod_{m \in \varsigma_{k} / j} I_{c_{m} \rightarrow u_{k}}^{t-1}\left(x_{j}\right)\right)\right\}
\end{gathered}
$$

Where, $t$ is the number of iterations, $\xi_{K}$ and $\zeta_{k}$ represents the non-zero position set of row $k$ and column $j$ in the $F$ matrix respectively.

Step 2: update the asset node:

$$
I_{u_{j} \rightarrow c_{k}}^{t}\left(x_{j}\right)=\prod_{m \in \xi_{j} / k} I_{u_{k} \rightarrow c_{m}}^{t}\left(x_{j}\right)
$$

Step 3: when formula (5) and formula (6) reach the maximum number of iterations, the output probability after MPA decoding $Q\left(x_{j}\right)$ is:

$$
Q\left(x_{j}\right)=\prod_{k \in \xi_{j}} I_{c_{k} \rightarrow u_{j}}^{t_{\max }}\left(x_{j}\right)
$$

By combining formula (4) - (7), we can get: 


$$
\begin{gathered}
I_{c_{k} \rightarrow u_{j}}^{t}\left(x_{j}\right)=\frac{1}{\sqrt{2 \pi} \delta} \times \max _{i=1,2, \ldots, \mathrm{N}}\left\{-\frac{1}{2 \delta^{2}}\left\|y_{k}-\sum_{v \in \varepsilon_{k}} h_{k, v} x_{k, v}\right\|^{2}+\sum_{v \in \varepsilon_{k}} I_{u_{j} \rightarrow c_{k}}^{t-1}\left(x_{j}\right)\right\} \\
I_{u_{j} \rightarrow c_{k}}^{t}\left(x_{j}\right)=\sum_{v \in \varepsilon_{k} / \mathrm{m}} I_{c_{m} \rightarrow u_{j}}^{t}\left(x_{j}\right)
\end{gathered}
$$

Where, $t$ still represents the number of iterations. When the algorithm passes the maximum number of iterations, the output probability of each user's codeword is:

$$
Q\left(x_{j}\right)=\sum_{v \in \varepsilon_{j}} I_{c_{k} \rightarrow u_{j}}^{t_{\max }}\left(x_{j}\right)
$$

\section{S-T-Max-log-MPA algorithm}

Based on the Max-log-MPA algorithm, the S-T-Max-log-MPA algorithm introduces the serial update algorithm and threshold MPA algorithm, in the Max-log-MPA algorithm, index (EXP) algorithm is changed into sum algorithm and maximum value. In the serial update algorithm, user node message update is integrated into resource node information update to reduce the complexity of information storage. In the threshold MPA algorithm, hard decision is used to effectively reduce the user node information that needs to be updated in each cycle. This algorithm combines the advantages of the three, can effectively reduce the complexity of the detection algorithm while maintaining a good bit error rate.

Because the S-T-Max-log-MPA algorithm first judges the stability of IoT users in the iterative update process, the formula (5) of the resource node update process of the algorithm is modified as follows:

$$
I_{c_{k} \rightarrow u_{j}}^{t}\left(x_{j}\right)=2 \times \frac{1}{\sqrt{2 \pi} \delta} \times \max _{\sim x_{j}}\left\{-\frac{1}{2 \delta^{2}}\left\|y_{k}-\sum_{v \in \xi_{k}} h_{k, v} x_{k, v}\right\|^{2} \times \prod_{m \in \mathcal{G}_{k} / j} I_{c_{m} \rightarrow u_{k}}^{t-1}\left(x_{j}\right)\right\}
$$

After that, the log likelihood ratio (LLR) of each user's coding bit determines the user:

$$
Q\left(x_{j}\right)=a p_{v}\left(x_{j}\right) \times \prod_{m \in \xi_{k} / j} I_{c_{m} \rightarrow u_{k}}\left(x_{j}\right)
$$

Where, $a p_{v}\left(x_{j}\right)$ represents the prior probability of the user $\mathrm{J}$ code word.

$$
\operatorname{LLR}_{j, x}=\log \left(\frac{P\left(b_{i}=0\right)}{P\left(b_{i}=1\right)}\right)=\log \left(\frac{\sum_{m: b_{m, i}=0} Q\left(x_{j}\right)}{\sum_{m: b_{m, i}=1} Q\left(x_{j}\right)}\right)
$$

Where, $L L R_{j, x}$ is the log likelihood ratio, $\sum_{m: b_{m, i}=0} Q\left(x_{j}\right)$ represents the output probability of the decoded variable node, $\sum_{m: b_{m, i}=1} Q\left(x_{j}\right)$ represents the output probability of the variable node to be decoded. $P\left(b_{i}=0\right)$ represents the probability of the decoded 
variable node $\mathrm{VN}$, and $P\left(b_{i}=1\right)$ represents the probability of the variable node $\mathrm{VN}$ to be decoded.

\section{Analysis of BER performance}

In the SCMA system, the T-Max-log-MPA algorithm combines the codeword credibility and user node stability, eliminates the exponential operation, and increases the judgment of the necessary conditions for the user node stability. Before the message is updated, the stability and threshold conditions of the user node are judged successively, which can not only increase the reliability of the decision codeword, but also reduce the loss of posterior soft information during the transmission process, which improves the accuracy of message delivery and BER performance. The S-T-Max-logMPA algorithm adds the serial mode to the T-Max-log-MPA algorithm. Because of the serial mode, the asynchronous mechanism is also added to the algorithm. Because of the existence of the asynchronous mechanism, in each iteration, all resource nodes can process and deliver messages at the same time, and the received messages can be delivered in a timely manner, which accelerates the convergence performance of the algorithm, and achieves the desired effect when the number of iterations is small, and further improve BER performance.

\section{Analysis of simulation results}

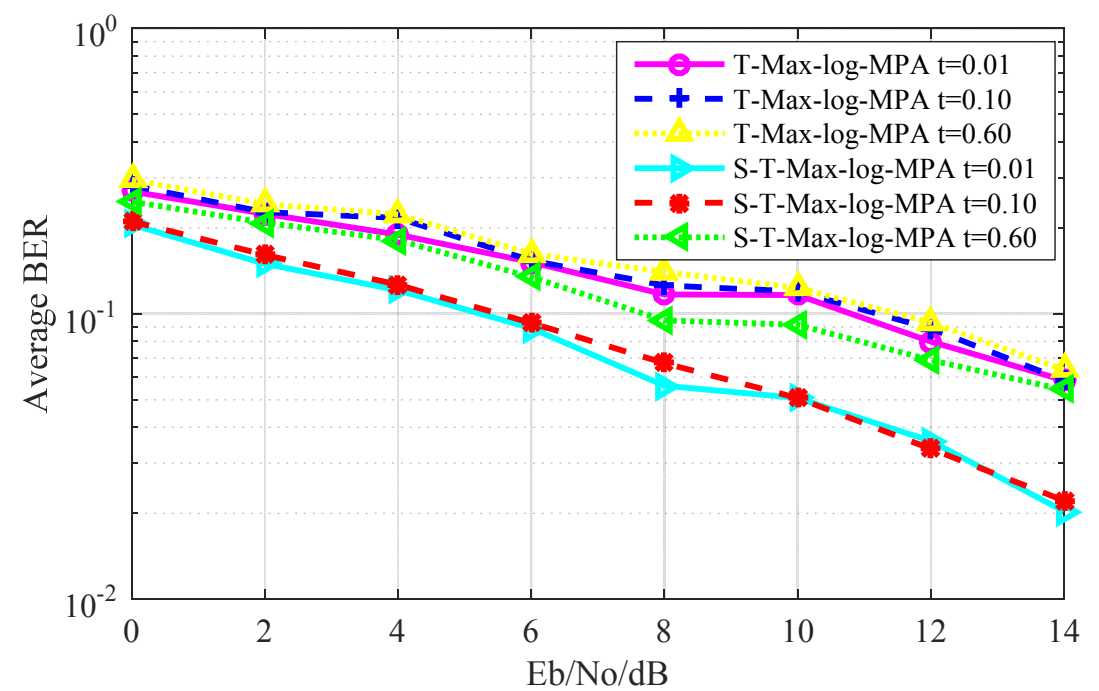

Figure 4 BER performance comparison between T-Max-log-MPA algorithm and S-T-Max-log-MPA algorithm when $\mathrm{T}_{\max }=2$

Figure 4 shows the BER performance comparison between T-Max-log-MPA algorithm and S-T-Max-log-MPA algorithm when $T_{\max }=2$. It can be seen from Figure 4 that when $\mathrm{T}_{\max }=2$, the overall BER performance of S-T-Max-log-MPA algorithm is 
higher than that of T-Max-log-MPA algorithm within the threshold value range of $0 \leq$ $\mathrm{E}_{\mathrm{b}} / \mathrm{N}_{\mathrm{o}} \leq 14 \mathrm{~dB}$, and the smaller the threshold, the more obvious the change, and the greater the BER performance difference. Comparing the BER performance of S-TMax-log-MPA and T-Max-log-MPA with the change of signal-to-noise ratio, we can see that when $\mathrm{E}_{\mathrm{b}} / \mathrm{N}_{\mathrm{o}}=0$, if the threshold value th $=0.01$, the BER of them are $20.55 \%$ and $26.83 \%$ respectively, and the BER of S-T-Max-log-MPA is $6.28 \%$ lower than that of T-Max-log-MPA. If the threshold value th $=0.10$, the BER performance of the two algorithms are $21.08 \%, 28.13 \%$ respectively, and the BER performance of S-T-Maxlog-MPA is $7.05 \%$ lower than that of T-Max-log-MPA. When the threshold value th= 0.6 , the BER performance of the two algorithms is $24.7 \%, 29.48 \%$ respectively, which is lower than $4.78 \%$ of T-Max-log-MPA algorithm. When $\mathrm{E}_{\mathrm{b}} / \mathrm{N}_{\mathrm{o}}=14$, calculate the BER when the thresholds are th $=0.01$, th $=0.1$, th $=0.6$ respectively. The BER performance of the S-T-Max-log-MPA algorithm under the above three thresholds are: $2.033 \%, 2.177 \%, 5.417 \%$, T-Max-log-MPA algorithm are 5.85\%, 5.85\%, $6.367 \%$, and their bit error rates differ by $3.817 \%, 3.673 \%, 0.959 \%$. It can be seen from the above that under the same threshold, with the increase of $\mathrm{E}_{\mathrm{b}} / \mathrm{N}_{\mathrm{o}}$, the smaller the bit error rate is, the better the optimization effect is. Therefore, when $\mathrm{T}_{\max }=2$, the performance of S-T-Max-log-MPA is better than that of T-Max-log-MPA.

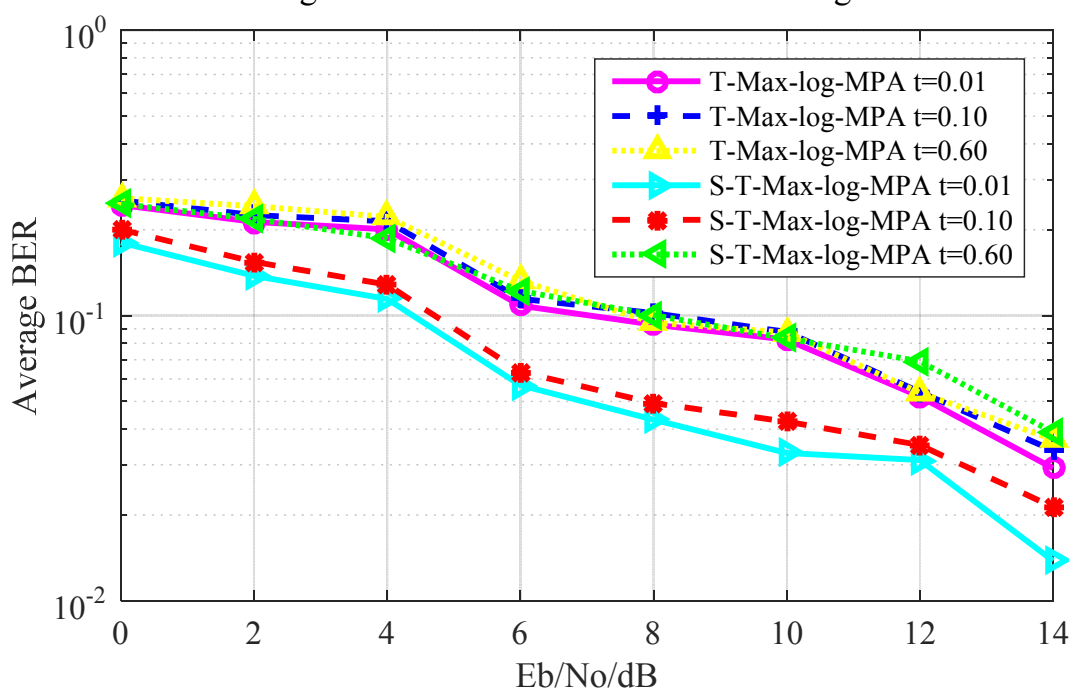

Figure 5 BER performance comparison between T-Max-log-MPA algorithm and S-T-Max-log-MPA algorithm when $\mathrm{T}_{\max }=3$

Figure 5 shows the performance comparison between S-T-Max-log-MPA algorithm and T-Max-log-MPA algorithm when $\mathrm{T}_{\max }=3$. When $\mathrm{E}_{\mathrm{b}} / \mathrm{N}_{\mathrm{o}}=0$, S-T-Max-log-MPA algorithm at the threshold value th $=0.01,0.1,0.6$, the corresponding BER performance is $18.02 \%, 20.03 \%, 24.58 \%$, T-Max-log-MPA algorithm corresponding values are $24.38 \%, 25.32 \%, 25.78 \%$, and the BER performance differs by $6.36 \%, 5.29 \%$, and $1.2 \%$. The performance of S-T-Max-log-MPA algorithm is best. When $\mathrm{E}_{\mathrm{b}} / \mathrm{N}_{\mathrm{o}}$ $=14$, S-T-Max-log-MPA algorithm at the threshold value th=0.01, 0.1, 0.6, the corresponding BER performance is $1.383 \%, 2.133 \%, 3.9 \%$, and T-Max-log-MPA algorithm corresponding values are $2.917 \%, 3.367 \%, 3.683 \%$. It can be seen from the 
results that when the threshold is less than 0.6, the performance of S-T-Max-log-MPA is better than that of T-Max-log-MPA. Therefore, when $T_{\max }=3$, the threshold value is less than 0.6, S-T-Max-log-MPA performance is better than T-Max-log-MPA.

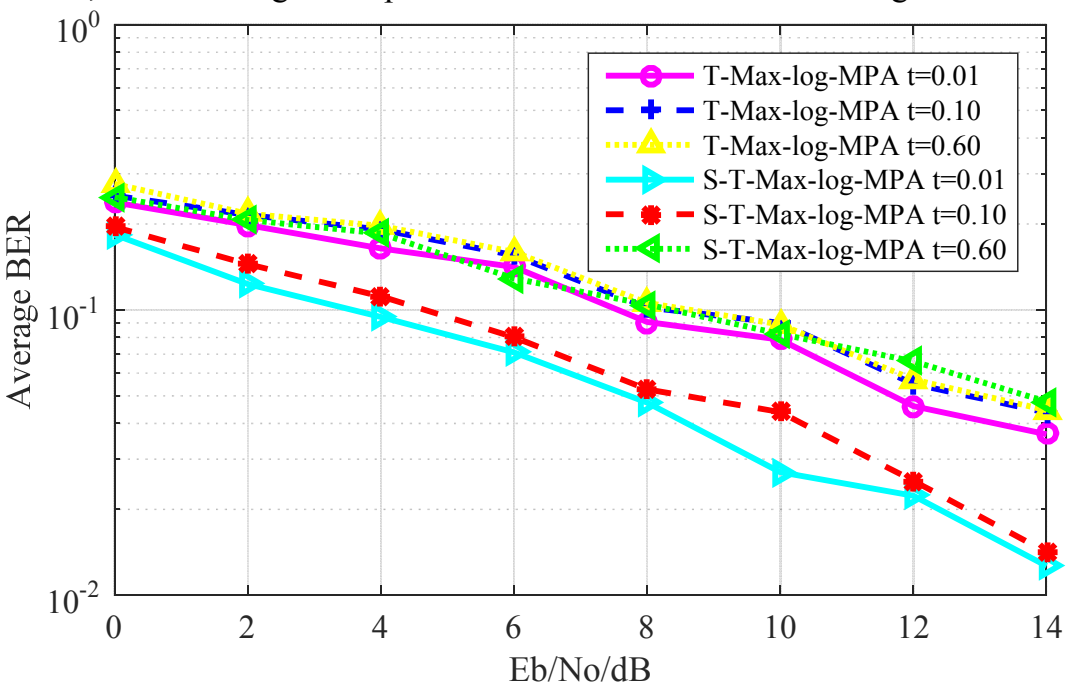

Figure 6 BER performance comparison between T-Max-log-MPA algorithm and S-T-Max-log-MPA algorithm when $\mathrm{T}_{\max }=5$

Figure 6 shows that when $\mathrm{T}_{\max }=5$ and the BER performance of S-T-Max-log-MPA algorithm and T-Max-log-MPA algorithm under the same threshold. As can be seen from the Figure 6, the BER performance of the S-T-Max-log-MPA algorithm is the best when the $\mathrm{E}_{\mathrm{b}} / \mathrm{N}_{\mathrm{o}}$ changes from 1 to 14 and th is less than 0.6. However, when th=0.6, the BER of S-T-Max-log-MPA algorithm is worse than T-Max-log-MPA algorithm, which indirectly shows that the best threshold value is less than 0.6 in the calculation process of the algorithm. Therefore, when selecting the optimal result, the part with threshold value th $<0.6$ is taken.

\section{Conclusion}

Based on the research of $5 \mathrm{G}$ based multi-user detection method in the IoT, this paper in-depth the S-T-Max-log-MPA algorithm and the T-Max-log-MPA algorithm. Through the comparison of experimental simulation, when the maximum number of iterations is 2,3 and 5, when the threshold value is less than 0.6 , with the change of $\mathrm{Eb} / \mathrm{No}$ of signal-to-noise ratio, the BER performance of S-T-Max-log-MPA algorithm is better than that of T-Max-log-MPA algorithm, and the smaller the threshold value, the more obvious the curve change, the more significant the BER performance improvement. It can be concluded that BER performance of S-T-Max-log-MPA algorithm is better than that of T-Max-log-MPA algorithm. The former algorithm can better solve the problem of poor BER performance of threshold messaging algorithm at low threshold, and can be better applied to multi-user detection based on 5G IoT. 
Funding: This work was supported by the Youth Science Foundation of Northeast Petroleum University under Grant No. 2019QNL-34 and Graduate Innovative Research Work and the National Natural Science Foundation of China under Grant (61871348).

Conflicts of Interest: The authors declare no conflict of interest.

\section{References}

1. Wan Peng. Research on NB-IoT terminal positioning technology [D]. Beijing University of Posts and telecommunications, 2018Author, F., Author, S.: Title of a proceedings paper. In: Editor, F., Editor, S. (eds.) CONFERENCE 2016, LNCS, vol. 9999, pp. 1-13. Springer, Heidelberg (2016).

2. Shah S H, Yaqoob I.[IEEE 2016 IEEE Smart Energy Grid Engineering (SEGE)-Oshawa, ON, Canada (2016.8.21-2016.8.24)] 2016 IEEE Smart Energy Grid Engineering (SEGE) - A survey: Internet of Things (IOT) technologies, applications and challenges[J]. 2016:381-385.Author, F.: Contribution title. In: 9th International Proceedings on Proceedings, pp. 1-2. Publisher, Location (2010).

3. Zhang Xiaoxu. Research on multi-user detection algorithm for M2M Communication [D]. University of Electronic Science and technology, may 2019

4. J.Boutros,E.Viterbo,C.Rastello,et.al, "Good Lattice Constellations for Both Rayleigh fading and Gaussian Channels," IEEE trans.On information theory,vol.42, issue 2,pp.502518,1996

5. Parvez Shaik,Praveen K. Singya,Vimal Bhatia. Performance analysis of QAM schemes for non-regenerative cooperative MIMO network with transmit antenna selection[J]. AEUE International Journal of Electronics and Communications,2019,107.

6. RAYMOND H Y L, YONGHUI L, BRANKA V, et al. Practical physical layer network coding for two-way relay channels: performance analysis and comparison[J].IEEE Transactions on Wireless Communications, 2011, 9(2): 764-777.

7. Taherzadeh M, Nikopour H, Bayesteh A, et.al. SCMA codebook design: proceedings of the 2014 IEEE 80th Vehicular Technology Conference (VTC2014-Fall), Vancouver, BC, Canada, September 14-17,2014[C].[S.1.]: IEEE,2014:1-5.

8. GAO Pengyu,Du Yang,DONG,Binhong, et al. LOW complexity CS-Aided MPA detector for SCMA systems [J]. IEEE Communications Letters, 2018, 22(4):784-787.

9. DU Y, DONG B, CHEN Z.Joint sparse graph-detector design for downlink MIMOSCMA system[J].IEEE Journals \& Magazines, 2017, 6(1):14-17.

10. H.F. Schepker, C. Bockelmann, A. Dekorsy. Exploiting sparsity in channel and data estimation for sporadic multi-user communication[C]. The Tenth International Symposium on Wireless.

11. Song Chunxue, Wen Ping, Zhang Xuechen. FPGA implementation of sparse code multiple access system based on $5 \mathrm{~g}$ wireless communication [J]. Application of electronic technology, 2016, 42 (7): 812 . 
12. Du Yang, Dong Binhong, Wang Xianjun, et al. SCMA multiuser detection algorithm based on serial strategy [J]. Journal of electronics and information, 2016, 38 (8): 16931888

13. MU H,MA Z,ALHAJI M,et al.A Fixed Low Complexity Message Pass Algorithm Detector for Up-link Scma System[J].IEEE Wireless Communications Letters,2015,4(6):585588.

14. Yang Du; Binhong Dong; Zhi Chen; Xiaodong Wang; Pengyu Gao. Improved Serial Scheduling-Based Detection for Sparse Code Multiple Access Systems, IEEE Wireless Communications Letters, Volume: Oct. 2017, 6, Issue: 5, pp: 570 - 573.

15. Lin Yang; Yunyun Liu; Yunming Siu. Low Complexity Message Passing Algorithm for SCMA System, IEEE Communications Letters, Dec. 2016, Volume: 20 , Issue: 12, pp: $2466-2469$.

16. Dacheng Wei; Yuxi Han ; Sihai Zhang; Lei Liu. Weighted message passing algorithm for SCMA, 2015 IEEE International Conference on Wireless Communications \& Signal Processing (WCSP), Nanjing, China,.15-17 Oct. 2015.

17. Guanghua Zhang, Zonglin Gu, Qiannan Zhao, Jingqiu Ren, weidang Lu. A ThresholdBased Max-log-MPA Low Complexity Multiuser Detection Algorithm, Sensors 2020, 20, 1016; doi:10.3390/s20041016.

18. Lei Lu,Yan Chen,Wenting Guo,Huilian Yang,Yiqun Wu,Shuangshuang Xing."Prototype for 5G new air interface technology SCMA and performance evaluation,"China Communications Dec 2015; Volume:12,Issue:Supplement,pp.38-48.

19. Shuai Han, Yiteng Huang, Weixiao Meng, Cheng Li, Nuo Xu and Dageng Chen. Optimal Power Allocation for SCMA Downlink Systems Based on Maximum Capacity, IEEE Transactions on Communications,Volume: 67 , Issue: 2, Page(s): 1480 - 1489.

20. Mahmoud Taherzadeh ; Hosein Nikopour ; Alireza Bayesteh ; Hadi Baligh." SCMA Codebook Design,” 2014 IEEE 80th Vehicular Technology Conference (VTC2014-Fall), 14-17 Sept. 2014, DOI: 10.1109/VTCFall.2014.6966170, Conference Location: Vancouver, BC, Canada

21. Jianjun Peng,Wei Chen,Bo Bai,Xin Guo, Chen Sun." Joint Optimization of Constellation With Mapping Matrix for SCMA Codebook Design," IEEE Signal Processing Letters,March 2017; Volume:24,Issue:3,pp.264-268.

22. Jing Xiaorong, Tao Hongbao. An optimal design method for sparse codebook multiple access codebook [J]. Journal of electronics and information, 2019,41 (01): 24-31.

23. Donghong Cai ; Pingzhi Fan ; Xianfu Lei ; Yingjie Liu ; Dageng Chen. Multi-Dimensional SCMA Codebook Design Based on Constellation Rotation and Interleaving, 2016 IEEE 83rd Vehicular Technology Conference (VTC Spring), Nanjing, China, 15-18 May 2016.

24. Mehmood Alam; Qi Zhang. Performance Study of SCMA Codebook Design, 2017 IEEE Wireless Communications and Networking Conference (WCNC), San Francisco, CA, USA, 19-22 March 2017.

25. Zhang Xu Ning, Ge Wen Ping, Liu Xi Teng. A partial codeword SCMA detection algorithm based on error compensation [J]. Chinese science and technology paper, 2019,14 (03): 268-272

26. Yang Wei, Zhao Yiwei, Hou Jianqi. An improved threshold based sparse code multiple access low complexity multiuser detection algorithm [J]. Journal of electronics and information, 2018,40 (05): 1044-1049 